\title{
Biological tools revamp disease classification
}

Just as different genes can contribute to similar diseases, so the same genes and families of genes can play a part in a range of different diseases. "The days when we had one gene, one disease have been dead for some time now," says Nicholas Katsanis of Johns Hopkins University School of Medicine in Baltimore, Maryland.

Scientists are using structural biology, genomics and biochemistry to reinvigorate a decadesold debate about the relationship between genes and disease. "If you have the genes and the mutations, you can now see how much of your previous disease classification system was real and how much was wrong," says clinical geneticist Han Brunner of Radboud University Nijmegen Medical Centre in the Netherlands. "The molecular biological information gives us a much finer view of what really is clinically important." It questions the idea that diseases with similar symptoms have the same genomic and biological origins.

A wave of studies is revealing the complicated relationship between genes and disease even in those disorders linked to glitches in a single gene. For example, a gene called $X P D$, which encodes a protein involved in DNA transcription and repair, has long intrigued scientists because different mutations in it cause three separate diseases. On 30 May, two separate teams published structures of XPD proteins in microorganisms $\mathrm{s}^{1,2}$, illuminating the causes behind XPD's 'three fates'.

The teams found that the XPD mutations causing one disease, xeroderma pigmentosum, damage the part of the XPD enzyme that binds DNA. As the mutated enzyme can't repair the DNA damage caused by sunlight, it explains why patients with this condition have such high rates of skin cancer. But they found that mutations causing trichothiodystrophy — marked by premature ageing — block the protein from joining in a larger complex that transcribes DNA. If the complex can't work, patients don't make enough protein, and their cells die young. But so might any incipient cancer cells, explaining why patients with this disease don't have the high cancer rates seen in those with xeroderma pigmentosum, the teams suggest.

But the XPD story has another layer of complexity: patients with the same underlying genetic mutations sometimes show different symptoms. That situation is familiar to human geneticists such as Brunner, who says that scientists should begin paying more attention to these supposed 'outliers'. "Just by accepting this as clinical variability, we are throwing away a lot of interesting biologically relevant information," he says.

In March, Katsanis's team published a paper on two diseases caused by malfunctioning cilia - tiny hairlike structures ubiquitous in the body. The paper ${ }^{3}$ showed that genes that cause one such disease, Meckel-Gruber syndrome, can also be mutated in patients with another ciliopathic disease, Bardet-Biedl syndrome. Katsanis's team further found that when patients carry mutations in genes associated with both diseases, they have unique symptoms not seen in either condition alone.

Katsanis's team went on to found that, indeed, genes linked to the two conditions interact with each other during development. Katsani proposes that the two conditions actually reflect disruptions in a single biological pathway. "Once we define functional modules containing multiple genes and look at disorders whose phenotypes fit into broad dysfunction of these modules, we're going to start seeing these phenomena time and time again," he says. Systems biologists are already attempting to define such 'functional modules' by drawing maps of connections between genes and diseases that have been found in association studies.

Although the price tag for genetic studies is dropping, clinicians' expertise remains expensive, and that worries Brunner. "Certainly with the amount of money people are spending genotyping thousands of patients, they are finding that the quality of the phenotype data is crucial," Brunner says. That's why, he says, a 'phenome project' is needed to investigate connections between phenotypes. The idea has been proposed before but stalled for lack of funding

\section{Erika Check Hayden}

\footnotetext{
1. Fan, L. et al. Cell 133, 789-800 (2008).

2. Liu, H. et. al. Cell 133, 801-812 (2008).

3. Leitch, C. C. et al. Nature Genet. 40, 443-448 (2008).
} 